\section{Novel Laboratory Exercises in Plant Tissue Culture: In Vitro Asymbiotic Germination of Orchid Seeds \\ Kenneth W. Mudge ${ }^{1}$ and Chin-Chang $\mathrm{Chu}^{2}$}

Additional index words. embryo culture, flasking, micropropagation, orchid, seed germination

Summary. In vitro asymbiotic seed germination, subculture, and outplanting of orchids is presented as a laboratory exercise suitable for students of plant propagation or tissue culture. Dendrobium antennatum (Lindley), Phalaenopsis (Blume) white hybrid, or both, are used in this exercise because they flower predictably in the greenhouse, are reliable for seed production, and germinate and grow rapidly in vitro. The exercises can be used to instruct students in the skills involved in orchid seed sterilization, sowing, and culture, as well as instruct students in the unique features of orchid reproductive biology and symbiosis. A schedule is suggested for stock plant flower pollination, capsule harvest, seed sowing, and seedling subculture so that the necessary plant material is available for students to sow, subculture, and outplant seedlings during a single laboratory session.

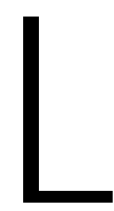

aboratory exercises designed to teach in vitro methods of propagation to students in introductory-level plant propagation or plant tissue culture courses naturally focus mainly on asexual (clonal) methods of in vitro culture, such as organogenesis, axillary shoot culture, and somatic embryogenesis. In vitro sexual propagation

Department of Floriculture and Ornamental Horticulture, 13 Plant Science Building, Cornell University, Ithaca, NY 14853. from seed is less commonly included as a student exercise, largely because it is relatively less-important commercially. Orchids are an important exception to this generalization. In vitro germination of orchid seeds, also known as flasking, is important in the commercial production of hybrid orchids and of many species such as Paphiopedilum sp. Pfitz. (Arditti and Ernst, 1993). In vitro asymbiotic germination is also an essential component of modern orchid breeding.

This exercise, presented along with sufficient background information via lecture or reading assignments (e.g., appropriateselections from Arditti, 1992; Arditti and Ernst, 1984; Hadley, 1982), can be used to teach the specific skills discussed below, but also to familiarize students with the unique historical, biological, and horticultural aspects of orchid reproduction. This exercise on in vitro asymbiotic orchid seed germination highlights an important event in the history of plant tissue culture, viz. Knudson's in vitro asymbiotic germination of CattleyaLindl. and Laelia Lindl. orchid seeds (Knudson, 1922), which was the first successful in vitro propagation of any plant.

Asymbiotic germination, as practiced in this exercise, can be contrasted to the unique natural symbiotic seed germination strategy that has evolved in the Orchidaceae. Specifically, sexual reproduction of orchids in nature involves germination and survival of a relatively small fraction of the large number of extremely small seeds produced within each fruit (capsule). In contrast to the seeds of most higher plant species, orchid seeds arequitesmall ( 0.4 to $1.25 \mathrm{~mm} \times 0.08$ to $0.27 \mathrm{~mm}$ ) (Aridtti, 1992), consisting of a seed coat and a rudimentary embryo that essentially lacks food reserves directly available to the embryo to support germination and early seedling development. Germination in nature is entirely dependent on the formation of a mycorrhizal association with an appropriate fungus and, hence, is referred to as symbiotic germination. By digesting soil organic matter such as cellulose, thefungus makes soluble sugars available to the embryo (Hadley, 1982). Aside from its obligate mycorrhizal association, embryo development in orchids differs from that of non-orchid higher plants in that, at the point of seed maturity, theorchid embryo is simply amoreor-less spherical mass of undifferentiated cells without a root-shoot axis. Upon imbibition and rupture of the testa, this proembryo enlarges and functions in the absorption of water and minerals from the substrate, and organic compounds from its fungal associate. It usually is called aprotocorm only after an apical shoot meristem differentiates, which occurs 4 to 5 weeks after sowing in the case of $D$. antennatum and the large-flowered Phalaenopsis hybrid used in this exercise. About 8 weeks after sowing, the first (adventitious) root meristem differentiates at the base of the first leaf (rather than from theprotocorm). The developmental sequence of seed germination for both Dendrobium antennatum and Phalaenopsis is illustrated in Fig. 1.

Knudson was able to bypass the natural requirementfor an appropriatemycorrhizal fungus and accomplish asymbiotic germination because he provided a simple sugar (sucrose) and other organic compounds in a tissue culture nutrient medium. In vitro orchid seed germination and early seedling development are obligately heterotrophic processes, in contrast to the facultative heterotrophic or photoautotrophic organic nutrition of non-orchid seedlings or other explants grown in vitro.

The student laboratory exercise described here includes three separate, but related, student activities, including: 1) sterilization and sowing of seed; 2) subculture of seedlings to fresh medium (2 months after sowing); and 3) outplanting of seedlings to the greenhouse ( 3 to 6 months after subculture). An additional necessary activity, which must be conducted in advance by the instructor, is flower pollination and harvesting of seed capsules. We stagger the various activities in order to have plant material at all three stages of development at onetime, so that all threestudent activities can be executed during a single laboratory session. A typical time line of instructor/student activities for this exercise for either Dendrobium antennatum or Phalaenopsis white hybrid is illustrated in Fig. 2. The requisite intervals between activities can vary, of course, depending on the choice of orchid species and on greenhouse and laboratory temperatures and, to some extent, illumination.

For this exercise, we have specifically chosen $D$. antennatumand Phalaenopsis white hybrid (any large-flowered Phalaenopsis hybrid is suitable) for several reasons. Using both species allows for acomparison of thetwo common orchid growth habits, i.e., Dendrobium is sympodial, whereas Phalaenopsis is monopodial (Fig. 1) (Arditti, 1992). Seeds of the former are larger than the latter. In vitro germination and seedling growth are relatively rapid for both. Dendrobium antennatum naturally blooms year round, while Phalaenopsis normally flowers three times per year in north-temperate North America, but the latter can be induced to flower at other times by exposing stock plants to $20 \mathrm{C}$ for 2 months followed by 2 to 3 months additional growing on at 25 to $30 \mathrm{C}$ (Lee and Lin, 1984). Self- or cross-pollination of either orchid can be done successfully.

For this student exercise, we use seed collected from a mature capsule, at or just prior to dehiscence. On the other hand, seeds of some orchid species, such as Cymbidium goeringii (Nagashima, 1993) and many interspecific Phalaenopsis hybrids germinate better if platedout before maturity. In such cases, a form of embryo (ovule) rescue ("green podding") commonly is practiced, in which an intact green seed capsule is surface-sterilized and immature seeds are extracted and placed in culture. An additional 


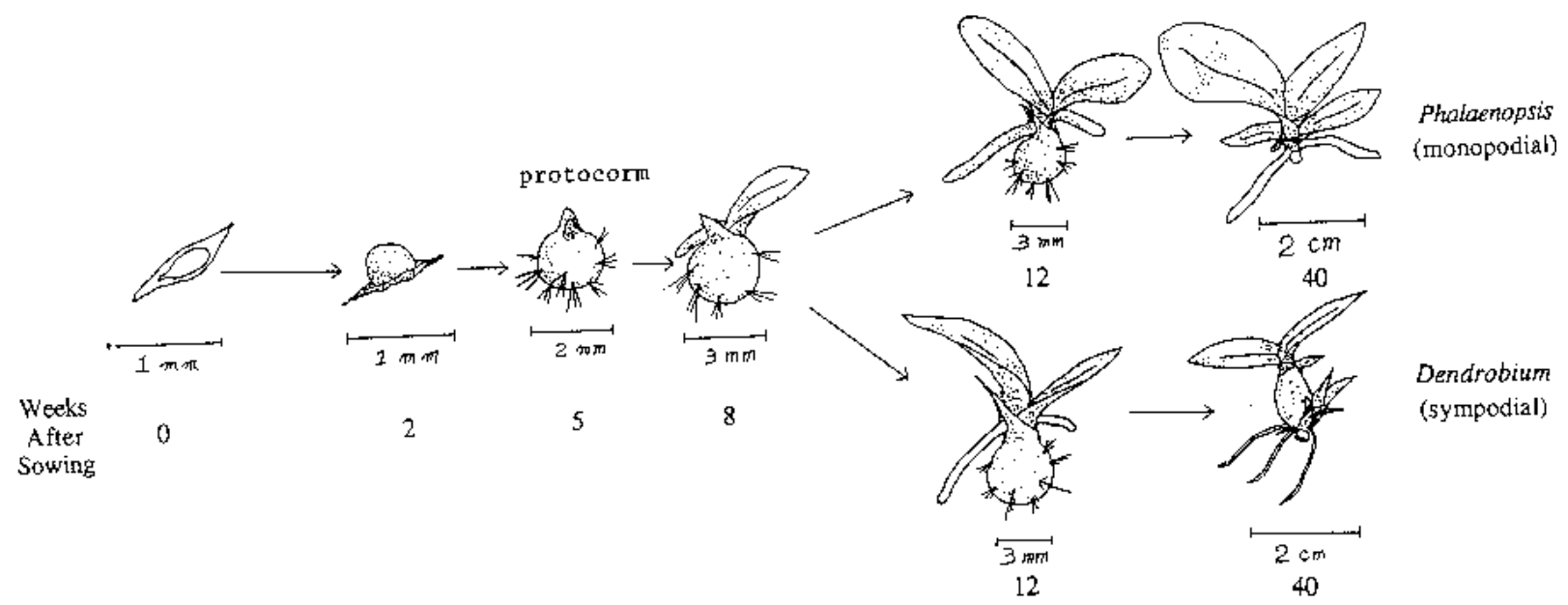

Fig. 1. Sequence of seed germination, protocorm and seedling development for Dendrobium antennatum and Phalaenopsis white hybrid.

advantage of "green pod" culture is that an intact capsuleis moreeasily surface-sterilized than loose mature seed. Nonetheless, in this exercise we prefer to use loose seeds collected from mature capsule for several reasons. Students learn the useful, but slightly more-challenging, technique involved in sterilization of loose seeds compared to an entire capsule. Fewer capsules and fewer stock plants are required because a single mature Phalaenopsis capsule, for example, will provide 50,000 to 100,000 seeds. The seeds from only one such capsule are sufficient for as many as 50 students, whereas at least one capsule per student would be required for "green pod" culture. Finally, mature seeds can bestored for 6 months or longer in a desiccator at room temperature, whereas immature seeds in or from green capsules lose viability rapidly.

\section{Seed sterilization and sowing}

Each student is given $\approx 1000$ ripe seeds, equivalent to $\approx 250 \mu$ loosely packed in a capped shell vial. Sterilization involves soaking seeds in a scintillation vial or a 50-ml Erlenmeyer flask containing $\approx 30 \mathrm{ml}$ of a solution of $0.5 \%$ sodium hypochlorite ( $w / v)$ plus one drop of Tween 20 . The solution should be swirled by hand about once each minute for 12 to $15 \mathrm{~min}$. Working under a laminar flow hood, sterile seeds are separated from the solution by filtering the contents of the flask, by vacuum or by gravity, through a presterilized filter paper disc in aBuchner or conical glass funnel. Seeds then are rinsed with sterile distilled water five or six times. A sterile inoculation loop is used to scrape seeds from the filter paper and streak them as evenly as possible onto the surface of the germination medium. The medium is Knudson C, as described by Arditti (1992), which is available commercially (K4003, Sigma, St. Louis), preformulated with $20 \mathrm{~g} \cdot$ liter $^{-1}$ sucrose. The $\mathrm{pH}$ is adjusted to 5.5 prior to addition of 7 $\mathrm{g} \cdot$ liter $^{-1}$ agar. After autoclaving, $20 \mathrm{ml}$ of this medium is poured into $9-\mathrm{cm}$ disposable plastic petri dishes. A sowing density of $\approx 300$ seeds per petri plate is appropriate, although seeds are too small to be counted accurately during sowing. Students are instructed to prepare two identical plates for each (or either) species. Petri plates should be sealed with Parafilm and placed in a culture room at $24 \mathrm{C}$ under illumination from coolwhite fluorescent tubes at about $70 \mu \mathrm{mol} \cdot \mathrm{m}^{-2} \cdot \mathrm{s}^{-1}$
PAR. Weekly or biweekly, over the next 8 weeks, students can observe germination through the petri dish lid by means of adissecting microscope. Typically, $95 \%$ of the seeds germinate. Students may be instructed to observe and sketch stages of development, as illustrated in Fig. 1.

\section{Subculture}

Using 8-week-old pregerminated seedlings, students, again working under a laminar flow hood, can transfer seedlings from germination plates to baby food jars or other relatively tall vessels containing amedium identical to that used for germination. Spacing between seedlings should be $\approx 15 \mathrm{~mm}$.

\section{Outplanting}

Three to 4.5 months after subculture, when seedlings have four fully developed leaves and are $\approx 5$ to $6 \mathrm{~cm}$ tall, they are ready for acclimatization and outplanting to the greenhouse. Good choices for the potting medium are either straight sphagnum moss or a mixture of four parts fine fir bark to onepart coarseperlite. Wetransplant 10 seedlings

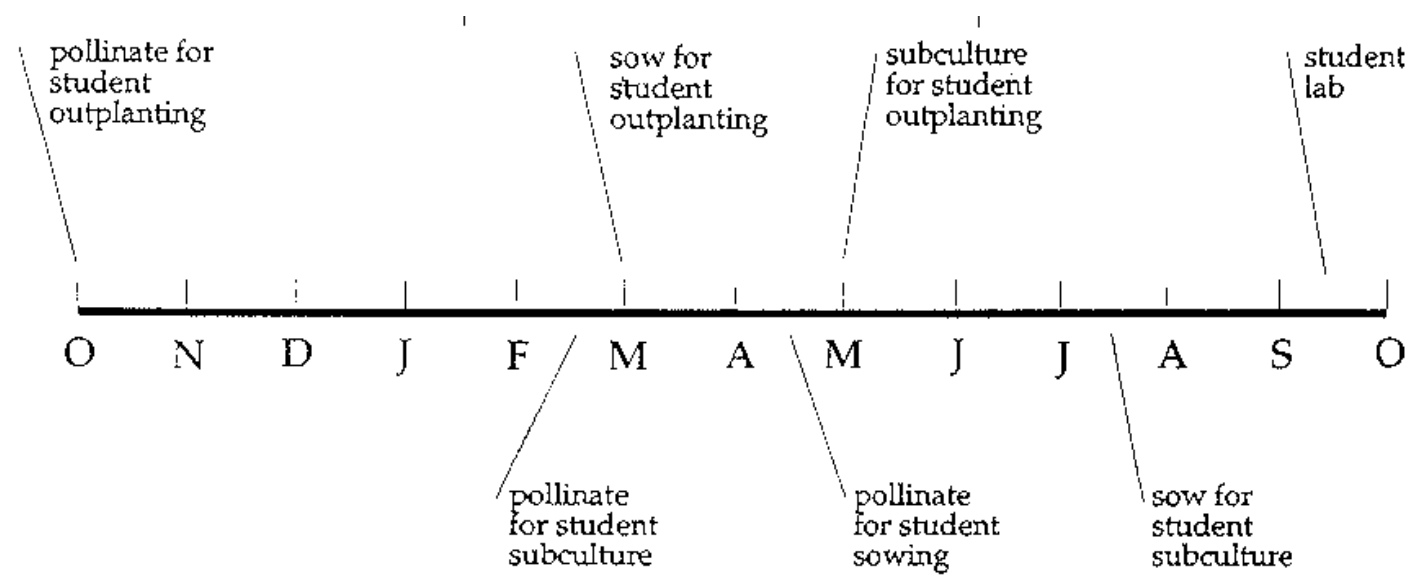

Fig. 2. Time line of instructor's preliminary activities required to have freshly harvested seed and in vitro seedlings at appropriate stages of development for student execution of all three laboratory activities (sowing, subculture, and outplanting) on a single date. 
into premoistened potting medium in a $10-\mathrm{cm}$ diameter plastic "community" pot. Immediately after outplanting, the pot is covered loosely with a plastic bag (humidity tent), the upper corners of which have been cut off diagonally to create two 1 inch long ventilation holes. Community pots are placed under $80 \%$ shade in a greenhouse at $24 \mathrm{C}$. Acclimatization is accomplished by gradually reducing the atmospheric relative humidity and increasing the light level. At the end of the first week in the greenhouse, the top edge of the plastic bag humidity tent can be cut off along its entire length. After 3 and 5 weeks, shading can be reduced to $70 \%$, and then $50 \%$, respectively, for Phalaenopsis, but Dendrobium antenatium can be lowered to, and permanently grown at, $30 \%$ shade.

We have found that many students who are training for a profession in horticulture or other aspects of plant science are especially curious about and interested in orchids. We have found this laboratory exercise involving orchids to be pedagogically useful because of the important principles and practices that it conveys, and also because it affords students the satisfaction of propagating and eventually taking hometheir very own orchids.

\section{Literature Cited}

Arditti., J. 1992. Fundamentals of orchid biology. Wiley, New York.

Arditti, J. and R. Ernst. 1984. Physiology of germinating orchid seeds. In: J. Arditti (ed.). Orchid biology: Reviews and perspectives. vol. III. Cornell Univ. Press, Ithaca, N.Y.

Arditti, J. and R. Ernst. 1993. Micropropagation of orchids. Wiley, New York.

Hadley, G. 1982. Orchid mycorrhiza. In: J. Arditti (ed.). Orchid biology: Reviews and perspectives. vol. II. Cornell Univ. Press. Ithaca, N.Y.

Knudson, L. 1922. Nonsymbiotic germination of orchid seeds. Bot. Gaz. 73:1-25.

Lee, N. and G. Lin. 1984. Effect of temperature on growth an flowering of Phalaenopsis white hybrid. J. Chinese Soc. Hort. Sci. 30:223-231.

Nagashima, T. 1993. Studies on the relationship between embryogenesis and germination in the Orchidaceae, J. Jpn. Soc. Hort. Sci. 62:581-594.

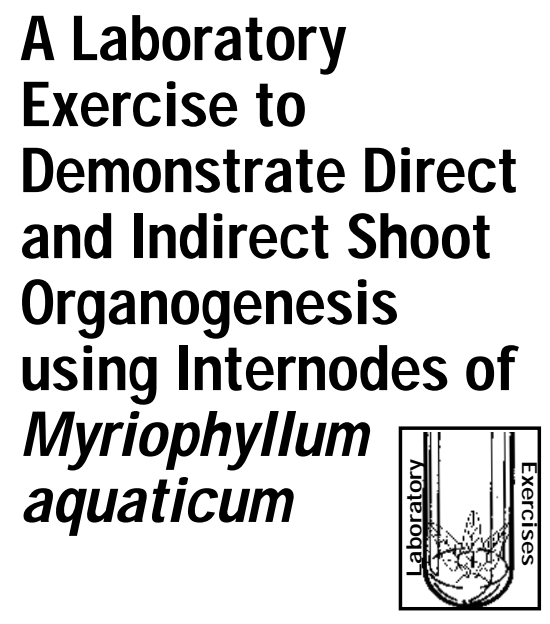

Michael E Kane, Nancy L. Philman, and Matthew A. Jenks

Additional index words. adventitious shoot formation, plant tissue culture, teaching, aquatic plant

Summary. Only a few plants are suitable for reliably demonstrating rapid direct and indirect shoot organogenesis in vitro. A laboratory exercise has been developed using internodes of Myriophyllum aquaticum, an amphibious water garden plant. Stock shoot cultures are established and maintained in vitro from nodal explants cultured on agar-solidified medium consisting of half-strength Murashige \& Skoog salts (MS) and $30 \mathrm{~g} \cdot$ liter $^{-1}$ sucrose. Students use these cultures as the source of internode explants. Explants are cultured on agar-solidified full-strength MS with 30 g.liter ${ }^{-1}$ sucrose, 100 mg.liter $^{-1}$ myo-inositol, and $0.4 \mathrm{mg}^{\circ}$ liter $^{-1}$ thiamine HCL and factorial combinations of 0 to $10 \mu \mathrm{M} 2 \mathrm{iP}$ and 0 to $1.0 \mu \mathrm{M} \mathrm{NAA}$. Adventitious shoot development occurs directly from the explant epidermis within 4 days and is promoted in media supplemented with $2 \mathrm{iP}$ alone. Oytokinin-supplemented media amended with NAA induce organogenetic callus formation, but reduce 2iP promotion of direct shoot organogenesis. After 4

Department of Environmental Horticulture, Institute of Food and Agricultural Science, University of Florida, Gainesville, FL 32611.

Florida Agricultural Experiment Station Journal Series no. R-03292. weeks, shoot organogenesis on the various media is quantified and can be analyzed statistically. Chemical names used: $N$-(3-methyl-2-butenyl)-1H-purin-6-amine (2iP); $\alpha$-naphthaleneacetic acid (NAA).

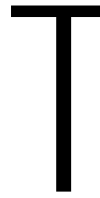

he most commonly used method for the micropropagation of most horticultural crops is shoot-tip culture. The primary feature of this method is that shoots are produced from shoot-tips or axillary buds that possess preexisting shoot meristems. Plants are produced following subsequent multiplication viaenhanced axillary branching and rooting of these shoots (Chu and Kurtz, 1990; George and Sherrington, 1984). Shoots also can be formed adventitiously from tissues through the process of shoot organogenesis. The two types of shoot organogenesis are indirect and direct shoot formation. Indirect shoot organogenesis involves the development of shoots indirectly from an intervening callus derived from the explant. Indirect shoot organogenesis may be less desirable for commercial clonal propagation because plants produced by this method may exhibit greater genetic variation (Cassells, 1985; Cassells and Morrish, 1987; Geier, 1991).

During direct shoot organogenesis, shoot meristems develop directly from the explant. Adventitious meristems arise from the epidermis or subjacent layer and usually are of single-cell origin. Direct shoot organogenesis is more desirable becausetheprobability of genetic variability among the plants produced is lower than with indirect organogenesis (Geier, 1991). However, one limitation to this method is that chimeras cannot be propagated true-to-type through production of adventitious shoots (Lineberger and Druckenbrod, 1985).

Plants such as Saintpaulia, Begonia, and Salpiglossiscan bepropagated readily in vitro by shoot organogenesis from pieces of internode, leaf blade, or petiole explants (Cooke, 1977; Lee et al., 1977; Start and Cumming, 1976; Takayama, 1983). For these genera, shoots can arise directly from the explant epidermis, but indirect shoot organogenesis also may occur if callus is promoted. Thelevels and combinations of cytokinin and auxins used can affect significantly the type of shoot organogenesis observed (Start and Cumming, 1976).

For commercial micropropagation, students must recognize the relationship between media selection, subsequent mode of plant regeneration, and resultant genetic stability of the plants produced. However, instructors encounter a dilemma when selecting laboratory exercises to demonstrate direct and indirect shoot organogenesis. Problems associated with poor surface sterilization success, extended culture duration, or sporadic shoot regeneration limit the usefulness of many species for teaching purposes. A reliable 